\title{
High-sensitive troponin $T$ increase after hemodialysis is associated with left ventricular global longitudinal strain and ultrafiltration rate
}

\author{
Serkan Ünlü̈ ${ }^{1,2,3}$, Asife Şahinarslan ${ }^{1}$, Burak Sezenöz ${ }^{1}$, Orhan Mecit Uludağ $\breve{g}^{3}$, \\ Gökhan Gökalp ${ }^{1}$, Özden Seçkin ${ }^{1}$, Selim Turgay Arınsoy ${ }^{4}$, \\ Özlem Gülbahar ${ }^{5}$, Nuri Bülent Boyacı ${ }^{1}$ \\ ${ }^{1}$ Department of Cardiology, Faculty of Medicine, Gazi University, Ankara, Turkey \\ ${ }^{2}$ Department of Cardiology, Atatürk Chest Diseases and Chest Surgery Education \\ and Research Hospital, Ankara, Turkey \\ ${ }^{3}$ Department of Pharmacology, Faculty of Pharmacy, Gazi University, Ankara, Turkey \\ ${ }^{4}$ Department of Nephrology, Faculty of Medicine, Gazi University, Ankara, Turkey \\ ${ }^{5}$ Department of Medical Biochemistry, Faculty of Medicine, Gazi University, Ankara, Turkey
}

\begin{abstract}
Background: Circulating troponin levels are both stable and higher in patients with end-stage renal disease, even in the absence of acute coronary syndrome. These patients commonly have underlying cardiac problems that frequently cause troponin elevation. The effect of hemodialysis (HD) on troponin levels has not been well elucidated. Thus, investigated herein is the relationship between the changes in troponin levels along with left ventricular deformation and volume depletion in patients with end-stage renal disease.

Methods: Patients included were between 18 and 85 years of age and were receiving hemodialysis for at least 6 months. High sensitive cardiac troponin T (hs-cTnT) levels were studied in blood samples taken at the beginning and end of HD. Two-dimensional speckle tracking strain imaging was used to evaluate myocardial contractility.

Results: Seventy patients $(50.7 \pm 16.9$ years of age, 27 women) were included in study. The mean volume of ultrafiltration was $3260 \pm 990 \mathrm{~mL}$. A significant increase in circulating hs-cTnT levels was observed, as well as a prominent decrease in left ventricular global longitudinal strain (GLS) after $H D(52.4 \pm 40.2 \mathrm{ng} / \mathrm{L}$ vs. $66.8 \pm 48.5 \mathrm{ng} / \mathrm{L}, p<0.001$ and $20.1 \pm 3.6 \%$ vs. $16.8 \pm 3.8 \% p<0.001$, respectively). Moreover, ultrafiltration rate and GLS were found as the strongest independent variables in relation to the relative increase in hs-cTnT.

Conclusions: Hemodialysis can cause a significant increase in hsTnT. This can jeopardize the accuracy of clinical diagnoses based on hs-TnT measurements. GLS may be used as a determinant of this hs-TnT increase. The influence of HD on the cardiovascular system should be kept in mind to prevent unnecessary interventions. (Cardiol J 2020; 27, 4: 376-383)
\end{abstract}

Key words: dialysis, high-sensitive, load, speckle, troponin

\section{Introduction}

Troponins (Tn) are structural proteins involved in the regulation of skeletal and cardiac muscle contractility and cardiac troponins (cTn) are being used as sensitive and specific markers of cardiac injury [1]. Recently developed, new generation, high-sensitive cardiac troponin (hs-cTn)

Address for correspondence: Serkan Ünlü, MD, MSc., Atatürk Chest Diseases and Chest Surgery Education and Research Hospital, Keçiören - Sanatoryum, Ankara, Turkey, tel: +905452472750, e-mail: unlu.serkan@gmail.com 
assays [2, 3] made it possible to detect cTn levels in lower concentrations and provided faster diagnostic information, which resulted in a reduced duration of patient hospitalization [4]. However, a variety of conditions can cause an increase in hscTn in the absence of ischemic heart disease [1], one of these conditions being, patients with endstage kidney disease who were shown to have higher circulating Tn levels compared to the normal population [5-12]. In these patients, underlying cardiac problems that frequently lead to cTn elevation are also common. However, the relationship between renal function disorders and the cTn increment is still not fully understood. The effect of dialysis on cTn levels has also not been robustly elucidated. Long-term cTn measurements in patients receiving dialysis therapy were shown to be higher than reference values without acute exacerbations $[7,8,12,13]$. There are, however, few studies examining the acute effect of dialysis on cTn levels. Moreover, end-stage renal disease increases the likelihood of having concomitant heart disease and structural abnormalities such as left ventricular (LV) hypertrophy which also causes electrocardiographic findings that mimic ischemia. Thus, clinicians are obliged to depend on Tn measurements to diagnose acute coronary syndromes. In addition, higher ultrafiltration volumes may result in myocardial stunning [14-16] and show an impact on cTn levels. LV global longitudinal strain (GLS) - a marker of LV systolic function - is associated with poor prognosis and has been suggested to be a better prognostic indicator than conventional $\mathrm{LV}$ ejection fraction (EF) in patients with chronic kidney disease $[17,18]$. Therefore, the present study was to investigate (i) the change in circulating hs-cTnT levels with hemodialysis (HD); (ii) the relationship between changes in hs-cTnT levels and volume depletion and (iii) changes in LV deformation as assessed by two-dimensional (2D) strain echocardiography with HD.

\section{Methods}

\section{Study population}

This study had a cohort of 70 patients, who were followed up in the Hemodialysis Unit of Gazi University Medical School Nephrology Department. Patients between 18 and 85 years of age, who were receiving systemic bicarbonate HD 3 times a week for at least 6 months were included. Patients with LV systolic dysfunction $(<53 \%)$ [19], evident coronary artery disease and moderate to severe valvular heart disease, atrial fibrillation, acute coronary syndrome, infectious and inflammatory conditions and pulmonary embolism were excluded from the study. This study was approved by the local ethical committee.

\section{Study protocol}

Blood samples and echocardiographic acquisitions were obtained by providing $72 \mathrm{~h}$ between $\mathrm{HD}$ sessions in order to achieve higher volume changes. Dry weight is targeted for each patient during HD sessions. Blood pressure, weight and heart rate were measured before and after HD. The ultrafiltration volume and rate were recorded.

\section{Blood samples}

Blood samples were collected immediately before and after HD. HsTnT levels were measured with a high sensitive troponin assay (Elecsys ${ }^{\circledR}$ Troponin T hs, Roche Diagnostics) with lower detection and upper reference $\left(99^{\text {th }}\right.$ percentile) limits of $3 \mathrm{ng} / \mathrm{L}$ and $14 \mathrm{ng} / \mathrm{L}$, respectively.

\section{Echocardiography}

All images were acquired and measurements were performed by an experienced sonographer (SÜ) with a GE Vivid 7 Dimension ultrasonography machine (GE Vingmed Ultrasound, Horten, Norway) equipped with a $3.5 \mathrm{MHz}$ transducer, immediately before and after HD. Electrocardiogram and respiration of patients were monitored. Echocardiographic images with at least three cardiac cycles were recorded at the end of expiration. The images were then transferred to a vendor-specific workstation and analysed with EchoPAC, BT 13 (GE Vingmed Ultrasound, Horten, Norway) software. All echocardiographic measurements were performed according to recent guidelines [19].

\section{$\mathrm{LV}$ strain and strain rate analysis}

Apical 4-, 3-, 2-chamber views were acquired with high frame rate ( $>60 \mathrm{fps}$ ) for $2 \mathrm{D}$ speckle tracking strain analysis. To define the region of interest, the endocardial surface was identified by manually placing at least 15 markings in all apical views. End-diastole was indicated by the peak of the R-wave on the electrocardiogram. Average GLS were measured as the mean of GLS from 4-, 3-, 2-chamber apical views. All strain and echocardiographic measurements were performed by SÜ, who has a long experience with deformation imaging. 


\section{Statistical analysis}

Continuous variables are presented as mean \pm standard deviation and categorical data are presented as percentages or frequencies. Kolmogorov-Smirnov was used to check for normality of distribution for continuous variables. The paired t-test and Wilcoxon test were used to compare parametric and nonparametric continuous variables, respectively, before and after HD. Categorical variables were compared by the $\chi^{2}$ test. The Pearson correlation test was used to evaluate the relationship among changes in hs-cTnT levels, GLS and fluid volume withdrawal. Univariate linear regression analysis with conventional clinical variables (age, gender etc.) and contributors with a significant correlation were entered in a multiple linear regression model. Possible collinearity was checked by using a tolerance and variance inflation factor. Variables with a tolerance of less than 0.10 and a variance inflation factor of 10 and above were withdrawn from the multivariate linear regression model. A two-tailed p-value of $<0.05$ was considered as statistically significant. All data were analysed with SPSS v.23.0.

\section{Results}

Among 80 patients screened, 70 of them were included in this study. Seven patients were excluded because of newly diagnosed LV systolic dysfunction, it was not possible to collect blood samples after HD in 3 patients. The baseline characteristics of participants are presented in Table 1. A decrease in both systolic and diastolic blood pressures and an increase in heart rate after HD was observed. However, no significant tachycardia (> $100 \mathrm{bpm}$ ) was detected after HD, except in 3 patients. The mean volume of ultrafiltration was $3260 \pm 990 \mathrm{~mL}$. Comparison of clinical vital signs before and after HD is shown in Table 2 .

A significant increase in serum hs-cTnT levels after HD was observed $(52.4 \pm 40.2 \mathrm{ng} / \mathrm{L}$ vs. $66.8 \pm$ $\pm 48.5 \mathrm{ng} / \mathrm{L}, \mathrm{p}<0.001$; Fig. 1 ). A relative change in hs-cTnT and ultrafiltration rate showed a significant correlation (Fig. 2).
The size of LV and left atrium decreased after HD, whereas LV EF remained unchanged. LV GLS also notably decreased after HD. Data of echocardiographic parameters are listed in Table 3. The relative change in GLS showed strong correlations with relative change in hs-cTnT (Fig. 3) and ultrafiltrated volume (Fig. 4).

In a linear regression analyses, relative change in GLS, ultrafiltration rate ultrafiltrated volume, and relative change in heart rate were significantly correlated with the relative change in hs-cTnT. Ultrafiltrated volume was removed from multivariate analysis since a high collinearity was found with ultrafiltration rate. Multivariate linear regression analysis revealed that the ultrafiltration rate and the relative change of LV GLS were the strongest independent variables associated with an increase in hs-cTnT levels (Table 4).

\section{Discussion}

In this study, the acute impact of HD on hscTnT levels were investigated. The main findings can be summarized as follows: (i) hs-cTnT levels

Table 1. Baseline characteristics of the participants.

\begin{tabular}{lc}
\hline Parameters & $\begin{array}{c}\text { Mean/Frequency } \\
\text { (n = 81) }\end{array}$ \\
\hline Age [year] & $50.7 \pm 16.9$ \\
Gender (male) & $43(61.4 \%)$ \\
BMI $\left[\mathrm{kg} / \mathrm{m}^{2}\right]$ & $23.5 \pm 4.4$ \\
Duration of HD [month] & $78.2 \pm 58.7$ \\
Ultra-filtrated volume $[\mathrm{mL}]$ & $3260 \pm 990$ \\
Ultrafiltration rate $[\mathrm{mL} / \mathrm{kg} / \mathrm{h}]$ & $11.6 \pm 3.0$ \\
Hypertension & $27(33.3 \%)$ \\
Hyperlipidemia & $24(29.6 \%)$ \\
Diabetes mellitus & $14(17.2 \%)$ \\
Glomerulonephritis & $10(12.3 \%)$ \\
Other & $13(16.1 \%)$ \\
Primary unknown renal failure & $7(8.6 \%)$ \\
\hline
\end{tabular}

$\mathrm{BMI}$ - body mass index; HD — hemodialysis; Other — polycystic kidney disease, amyloidosis, nephrolithiasis, vesicoureteral reflux, pyelonephritis, autoimmune diseases, toxic nephropathy

Table 2. Vital clinical parameters before and after hemodialysis (HD).

\begin{tabular}{lccc}
\hline Parameters & Before HD & After HD & P \\
\hline Systolic blood pressure $[\mathrm{mmHg}]$ & $123.3 \pm 21.7$ & $104.2 \pm 21.6$ & $<0.001$ \\
Diastolic blood pressure $[\mathrm{mmHg}]$ & $74.4 \pm 12.9$ & $62.6 \pm 13.1$ & $<0.001$ \\
Heart rate $[\mathrm{bpm}]$ & $78 \pm 15$ & $86 \pm 16$ & $<0.001$ \\
Weight $[\mathrm{kg}]$ & $70.4 \pm 17.6$ & $66.6 \pm 15.2$ & $<0.001$ \\
\hline
\end{tabular}




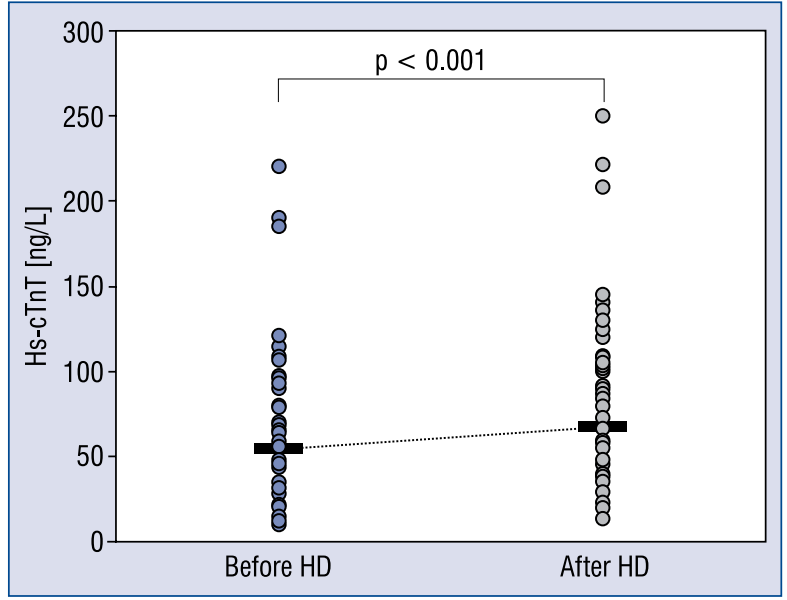

Figure 1. High-sensitive cardiac troponin T (hs-cTnT) levels before and after hemodialysis (HD). Comparison between mean values before and after HD is presented. Significance is indicated on the graph.

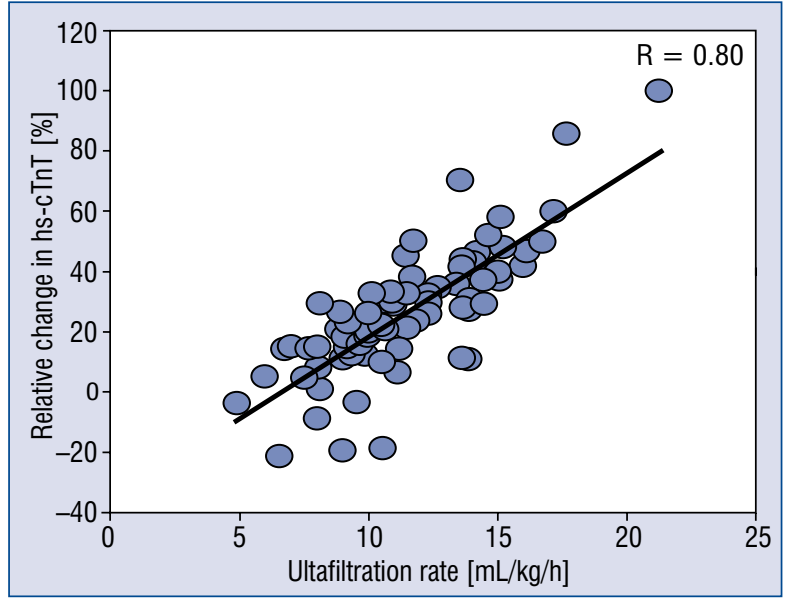

Figure 2. Comparison between relative change of highsensitive cardiac troponin $\mathrm{T}$ (hs-cTnT) (y-axis) and the ultrafiltration rate ( $x$-axis). The correlation coefficient $(R)$ is indicated. $P$ value is $<0.001$.

Table 3. Echocardiographic measurements before and after hemodialysis (HD).

\begin{tabular}{|c|c|c|c|}
\hline Parameters & Before HD & After HD & $\mathbf{P}$ \\
\hline \multicolumn{4}{|c|}{ Dimensions. areas and volumes of left heart } \\
\hline LV end-diastolic diameter $(\mathrm{cm})$ & $4.4 \pm 0.7$ & $4.0 \pm 0.8$ & $<0.001$ \\
\hline LV end-diastolic volume (ml) & $75.3 \pm 22.7$ & $68.5 \pm 29.7$ & $<0.001$ \\
\hline LV end-systolic volume [mL] & $26 \pm 11.2$ & $23.8 \pm 17.8$ & 0.401 \\
\hline LV ejection fraction [\%] & $66.0 \pm 11.5$ & $64.2 \pm 10.6$ & 0.071 \\
\hline LA diameter $[\mathrm{cm}]$ & $5.1 \pm 1.4$ & $4.7 \pm 1.4$ & $<0.001$ \\
\hline LA area $\left[\mathrm{cm}^{2}\right]$ & $29.9 \pm 10.6$ & $19.9 \pm 10.3$ & $<0.001$ \\
\hline LA volume index $\left[\mathrm{mL} / \mathrm{m}^{2}\right]$ & $48.7 \pm 16.3$ & $33.4 \pm 16.1$ & $<0.001$ \\
\hline \multicolumn{4}{|c|}{ Doppler measurements of left ventricle } \\
\hline $\mathrm{E}[\mathrm{cm} / \mathrm{s}]$ & $99 \pm 24.8$ & $65.5 \pm 23.1$ & $<0.001$ \\
\hline$A[\mathrm{~cm} / \mathrm{s}]$ & $94 \pm 92.1$ & $76.7 \pm 26.9$ & $<0.001$ \\
\hline$E / A$ & $1.18 \pm 0.6$ & $0.92 \pm 0.34$ & $<0.001$ \\
\hline \multicolumn{4}{|c|}{ Tissue Doppler measurements of left ventricle } \\
\hline $\mathrm{E}_{\text {lateral }}^{\prime}[\mathrm{cm} / \mathrm{s}]$ & $11.1 \pm 2.8$ & $10.1 \pm 2.8$ & 0.001 \\
\hline $\mathrm{A}_{\text {lateral }}^{\prime}[\mathrm{cm} / \mathrm{s}]$ & $12.5 \pm 4.1$ & $10.2 \pm 3.4$ & $<0.001$ \\
\hline $\mathrm{S}_{\text {lateral }}^{\prime}[\mathrm{cm} / \mathrm{s}]$ & $11.1 \pm 10.2$ & $9.6 \pm 2.9$ & $<0.001$ \\
\hline$E / E_{\text {lateral }}^{\prime}$ & $8.8 \pm 3.8$ & $6.3 \pm 2.9$ & $<0.001$ \\
\hline $\mathrm{E}_{\text {septal }}^{\prime}[\mathrm{cm} / \mathrm{s}]$ & $10.9 \pm 2.5$ & $10.1 \pm 2.8$ & 0.001 \\
\hline $\mathrm{A}_{\text {septal }}^{\prime}[\mathrm{cm} / \mathrm{s}]$ & $8.8 \pm 2$ & $7.9 \pm 2.4$ & $<0.001$ \\
\hline $\mathrm{S}_{\text {septal }}^{\prime}[\mathrm{cm} / \mathrm{s}]$ & $10 \pm 3.6$ & $8 \pm 2.6$ & $<0.001$ \\
\hline$E / E_{\text {septal }}^{\prime}$ & $9.6 \pm 3.7$ & $8.0 \pm 2.4$ & $<0.001$ \\
\hline \multicolumn{4}{|c|}{ Speckle tracking echocardiography } \\
\hline LV GLS [\%] & $-20.1 \pm 3.6$ & $-16.8 \pm 3.8$ & $<0.001$ \\
\hline
\end{tabular}

GLS - global longitudinal strain; LA — left atrium; LV — left ventricle 


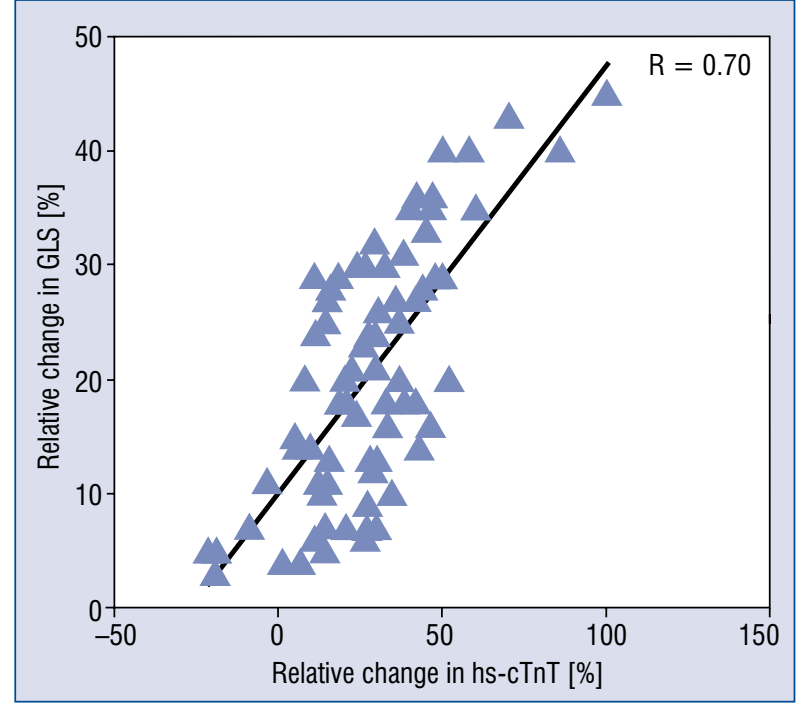

Figure 3. Comparison between relative change of global longitudinal strain (GLS) measurements (y-axis) and relative change in high-sensitive cardiac troponin T (hs-cTnT) (x-axis). The correlation coefficient $(R)$ is indicated. $P$ value is $<0.001$.

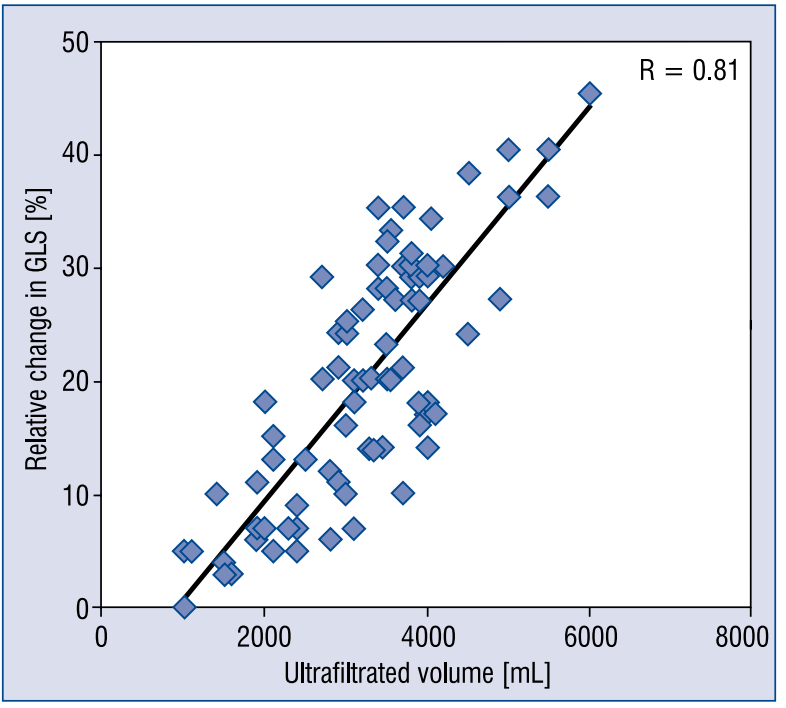

Figure 4. Comparison between relative change of global longitudinal strain (GLS) measurements (y-axis) and the ultrafiltrated volume (x-axis). The correlation coefficient $(R)$ is indicated. $P$ value is $<0.001$.

Table 4. Determinants of relative increase in high sensitive troponin T levels after hemodialysis.

\begin{tabular}{lccccc}
\hline Parameters & \multicolumn{2}{c}{ Univariate } & & \multicolumn{3}{c}{ Multivariate } \\
\cline { 2 - 3 } & $\mathbf{r}$ & $\mathbf{p}$ & & $\mathbf{R}^{2}$ & $\mathbf{p}$ \\
\hline Age [year] & -0.200 & 0.159 & & \\
Gender & -0.187 & 0.282 & & \\
Change in systolic BP & 0.386 & 0.10 & & \\
Change in diastolic BP & 0.248 & 0.147 & & 0.001 \\
Change in heart rate & 0.49 & 0.045 & & \\
Change in LV GLS & 0.70 & $<0.0001$ & & $<0.0001$ \\
Change in LV EDV & 0.350 & 0.106 & & \\
Ultrafiltration rate $[\mathrm{mL} / \mathrm{kg} / \mathrm{h}]$ & 0.80 & $<0.0001$ & & \\
\hline
\end{tabular}

BP — blood pressure; EDV — end-systolic volume; GLS - global longitudinal strain; LV — left ventricle

show a statistically significant increase after HD, (ii) relative change in hs-cTnT levels shows strong correlation with ultrafiltration rate, (iii) LV systolic function evaluated by 2D speckle tracking analysis deteriorates with high rapid volume depletion, (iv) the relative change in GLS and ultrafiltration rate are independently associated with a relative hscTnT increase after HD.

\section{Selection of study population}

There have been several studies which aimed to investigate the impact of HD on cTn levels $[7,8,10-13,20-23]$ According to available research, none of the studies evaluated the relation of ultrafiltration rate, deformation indices and changes in circulating cTn levels. Therefore, it was decided to investigate patients with end-stage kidney disease at a time when the highest volume change can be achieved. By having $3200 \mathrm{~mL}$ of average ultrafiltration volume, it was believed that this would obtain the highest possible volume changes in the literature. In addition, patients without significant cardiac diseases, except LV hypertrophy, were enrolled to avoid possible effects of cardiac pathologies on changes in cTn levels.

\section{Changes in vital signs and} cardiac function with HD

In the present study, both systolic and diastolic blood pressures decreased after HD, which is 
a physiologic and expected response to a loss of fluid and modest decrease in afterload. The heart rate of subjects studied increased after HD as a physiologic response to reduced circulating volume.

\section{Impact of $\mathrm{HD}$ on circulating hs-cTn levels}

In present study, an almost $30 \%$ relative increase in hs-cTn levels was found. In available literature, conflicting results have been presented about cTn change with $\mathrm{HD}[7,8,10-13,20-22]$. The current perception is to expect a modest relative decrease in circulating cTn levels after HD [13]. However, the rate of ultrafiltrated volume could also have an impact on cTn change. The current study showed that ultrafiltrated volume showed a strong correlation with the relative change in hs-cTn levels.

Elevated cTn concentrations after HD may reflect increased concentration due to reduced clearance with limited residual renal function [24]. Recurrent myocardial damage could be a potent reason for cTn elevation [15]. Since the present population had a mean of almost 7 years for receiving HD, chronicity of renal replacement therapy could have caused cardiac and renal structural damage which could lead to a cTn increase in each session of HD. Moreover, it has been claimed that HD can lead to myocardial stunning especially in high ultrafiltration rates which can also lead to acute elevation of hs-cTn T levels [14, 25]. Since these patients are at greater risk of coronary artery disease development, high volume depletion may cause reflex tachycardia and ischemia as a result of supply-demand mismatch during HD sessions, which may contribute to cTn elevation as well as LV hypertrophy.

\section{Impact of HD on echocardiographic parameters}

Although LV and left atrial dimensions, areas and volumes were significantly reduced, no significant change in LV EF was observed. Since EF is a relative measurement, volume change is not expected to affect $\mathrm{EF}$ measurements in individuals with normal systolic function. Nonetheless, there are conflicting findings about LV EF change with volume depletion evidenced in research literature, which could be due to differing sample sizes and methodologies of the studies.

Two-dimensional speckle tracking is a method developed for functional assessment of LV. 2D speckle tracking derived GLS has now emerged as a new index of LV function [19, 26]. GLS appears to be robust and more sensitive for detection of subtle functional changes, providing complementary and superior information over conventional LV EF measurements [27, 28]. In the present study, a significant reduction in LV GLS by being highly affected from volume change was found. Myocardial stunning could also lead to a decrease in LV GLS by having an impact on myocardial contractility. LV systolic function evaluated with GLS during and after HD may present cardiac systolic dysfunction. Thus patients should be closely monitored and clinicians should be aware of possible transient, cardiac decompensation, considering concomitant hs-cTn elevation.

\section{Clinical perspective of hs-cTnT increase after HD}

Chest pain is the most common cause of visits to emergency department and almost one-quarter of these patients are diagnosed with acute coronary syndrome $[1,4]$. To exclude acute myocardial infarction in patients with acute onset of chest pain, 6-12 h of follow-up was needed with older generations of cTn assays [4, 29]. It was therefore raising a significant burden on the health care system and the possibility of developing faster diagnostic algorithms would be a great advantage [4]. Patients with end stage kidney disease have increased circulating cTn levels. In the present population cTn levels were shown to be high but stable compared to reference values. However, conflicting findings are present on the acute effect of HD on cTn levels [8, 13, 24, 30]. Nevertheless, clinicians must mainly rely on cTn measurements since patients with chronic kidney disease also have the most cardiovascular risk factors and ischemic findings on electrocardiography which could be present due to LV hypertrophy. High blood pressure, hyperdynamic circulation due to arteriovenous shunt, volume overload and increased pulse pressure can cause myocardial strain and result in elevated cTn in this population $[14,15$, 25]. Moreover, recurrent myocardial damage due to decreased myocardial blood flow caused by HD plays an important role in cardiac remodeling [14, 16]. Thus, high ultrafiltration rates and volumes are especially shown to be associated with myocardial stunning [25]. Herein an almost 30\% relative increase in hs-cTnT levels with HD and it was demonstrated that this increase is highly correlated with the ultrafiltration rate. It was also shown that high ultrafiltration volumes have a substantial impact on systolic function of LV. Relative change in LV GLS also showed a very strong correlation with the ultrafiltrated rate. Moreover, LV GLS was found to be one of the strongest independent variables in relation to a relative hs-cTnT increase. 
Therefore, it can be claimed that the rate of ultrafiltration has a substantial impact on hs-cTnT change and myocardial contractility which can be also related to an hs-cTnT increase. As suggested in the recent guidelines [25], ultrafiltration rates and volumes should be minimized to suit what is best for patients to achieve hemodynamic stability and tolerability. Additional HD sessions should be considered for patients instead of extensive ultrafiltration rates [25]. Patients on HD therapy presenting with chest pain must still be managed by measuring serial $\mathrm{c} T n$ levels, the change in $\mathrm{cTn}$ may help in clinical decision making as to whether it is acute coronary syndrome or not.

\section{Limitations of the study}

In this present study it was endeavoured to enrol an adequate number of patients to represent a population with end-stage kidney disease. There was no possibility of telemetric monitoring or obtaining electrocardiogram records which may have played an additional role on possible causes of c $\mathrm{Tn}$ elevation before and after HD, however it was not needed clinically since none of subjects had related symptoms. Since patients with coronary artery disease were excluded and the present population included patients without previous coronary angiogram, an inability to evaluate the possible role of significant coronary stenosis causing ischemia in these patients might have interfered with the results. The aim of this study was to investigate the acute effects of HD on hs-cTnT levels and its relation with ultrafiltration rate and therefore, hs-c TnT measurements on $12^{\text {th }}$ or $24^{\text {th }} \mathrm{h}$ after HD could not be presented. Moreover, HD has various effects on cardiovascular dynamics, although we found ultrafiltration rate and LV GLS as independent variables related to a relative increase of hs-cTnT, exact mechanisms of this increase in hs-cTnT level require further investigation and cannot be quintessentially elucidated with the present findings. To avoid possible variability in measurements of echocardiographic parameters, a single observer (SÜ), who has substantial experience in echocardiography and deformation imaging, performed all echocardiographic analyses of the image datasets.

\section{Conclusions}

In the present study, it was shown that hs-cTnT levels show a significant increase after HD which can jeopardize the accuracy of clinical diagnoses based on hs-cTnT measurements. A strong correlation was present among relative changes in
hs-cTnT levels, LV GLS, and ultrafiltration rate. LV GLS was found to be one of the strongest independent variables associated with the relative hs-cTnT increase. Rapid volume depletion substantially effected myocardial contractility and resulted in possible myocardial damage. The rate of ultrafiltration should be minimized to avoid possible negative consequences. Considering the effect of HD with high ultrafiltration rate on circulating hs-cTnT levels, it could be of use to prevent misdiagnosis.

\section{Conflict of interest: None declared}

\section{References}

1. Thygesen K, Alpert J, Jaffe A, et al. Third universal definition of myocardial infarction. Nat Rev Cardiol. 2012; 9(11): 620-633, doi:10.1038/nrcardio.2012.122.

2. Westermann D, Neumann JT, Sörensen NA, et al. High-sensitivity assays for troponin in patients with cardiac disease. Nat Rev Cardiol. 2017; 14(8): 472-483, doi: 10.1038/nrcardio.2017.48, indexed in Pubmed: 28383022.

3. Apple FS, Collinson PO. Analytical characteristics of high-sensitivity cardiac troponin assays. Clin Chem. 2012; 58(1): 54-61, doi: 10.1373/clinchem.2011.165795, indexed in Pubmed: 21965555.

4. Roffi M, Patrono C, Collet JP, et al. 2015 ESC Guidelines for the management of acute coronary syndromes in patients presenting without persistent ST-segment elevation: Task Force for the Management of Acute Coronary Syndromes in Patients Presenting without Persistent ST-Segment Elevation of the European Society of Cardiology (ESC). Eur Heart J. 2016; 37(3): 267-315, doi: 10.1093/eurheartj/ehv320, indexed in Pubmed: 26320110.

5. Hassan HC, Howlin K, Jefferys A, et al. High-sensitivity troponin as a predictor of cardiac events and mortality in the stable dialysis population. Clin Chem. 2014; 60(2): 389-398, doi: 10.1373/ /clinchem.2013.207142, indexed in Pubmed: 24185551.

6. Mishra RK, Li Y, DeFilippi C, et al. CRIC Study Investigators. Association of cardiac troponin $\mathrm{T}$ with left ventricular structure and function in CKD. Am J Kidney Dis. 2013; 61(5): 701-709, doi: 10.1053/j.ajkd.2012.11.034, indexed in Pubmed: 23291148.

7. Pianta TJ, Horvath AR, Ellis VM, et al. Cardiac high-sensitivity troponin $\mathrm{T}$ measurement: a layer of complexity in managing haemodialysis patients. Nephrology (Carlton). 2012; 17(7): 636-641, doi: 10.1111/j.1440-1797.2012.01625.x, indexed in Pubmed: 22694299.

8. Kumar N, Michelis MF, DeVita MV, et al. Troponin I levels in asymptomatic patients on haemodialysis using a high-sensitivity assay. Nephrol Dial Transplant. 2011; 26(2): 665-670, doi: 10.1093/ndt/gfq442, indexed in Pubmed: 20656755.

9. deFilippi C, Wasserman S, Rosanio S, et al. Cardiac troponin T and $\mathrm{C}$-reactive protein for predicting prognosis, coronary atherosclerosis, and cardiomyopathy in patients undergoing longterm hemodialysis. JAMA. 2003; 290(3): 353-359, doi: 10.1001/ /jama.290.3.353, indexed in Pubmed: 12865376.

10. Yakupoglu U, Ozdemir FN, Arat Z, et al. Can troponin-I predict cardiovascular mortality due to myocardial injury in hemodialysis patients? Transplant Proc. 2002; 34(6): 2033-2034, indexed in Pubmed: 12270303. 
11. Apple FS, Murakami MM, Pearce LA, et al. Predictive value of cardiac troponin I and T for subsequent death in end-stage renal disease. Circulation. 2002; 106(23): 2941-2945, indexed in Pubmed: 12460876.

12. Fredericks $\mathrm{S}$, Chang $\mathrm{R}$, Gregson $\mathrm{H}$, et al. Circulating cardiac troponin- $\mathrm{T}$ in patients before and after renal transplantation. Clin Chim Acta. 2001; 310(2): 199-203, indexed in Pubmed: 11498086 .

13. Chen M, Gerson H, Eintracht S, et al. Effect of Hemodialysis on Levels of High-Sensitivity Cardiac Troponin T. Am J Cardiol. 2017; 120(11): 2061-2064, doi: 10.1016/j.amjcard.2017.08.026, indexed in Pubmed: 29033047.

14. Dasselaar JJ, Slart RH, Knip M, et al. Haemodialysis is associated with a pronounced fall in myocardial perfusion. Nephrol Dial Transplant. 2009; 24(2): 604-610, doi: 10.1093/ndt/gfn501, indexed in Pubmed: 18775808.

15. Burton JO, Jefferies HJ, Selby NM, et al. Hemodialysis-induced cardiac injury: determinants and associated outcomes. Clin J Am Soc Nephrol. 2009; 4(5): 914-920, doi: 10.2215/CJN.03900808, indexed in Pubmed: 19357245.

16. Bemelmans RHH, Boerma EC, Barendregt J, et al. Changes in the volume status of haemodialysis patients are reflected in sublingual microvascular perfusion. Nephrol Dial Transplant. 2009; 24(11): 3487-3492, doi: 10.1093/ndt/gfp267, indexed in Pubmed: 19515801.

17. Hensen LCR, Goossens K, Delgado V, et al. Prognostic implications of left ventricular global longitudinal strain in predialysis and dialysis patients. Am J Cardiol. 2017; 120(3): 500-504, doi: 10.1016/j.amjcard.2017.04.054, indexed in Pubmed: 28579125 .

18. Krishnasamy R, Isbel NM, Hawley CM, et al. Left ventricular global longitudinal strain (GLS) is a superior predictor of allcause and cardiovascular mortality when compared to ejection fraction in advanced chronic kidney disease. PLoS One. 2015; 10(5): e0127044, doi: 10.1371/journal.pone.0127044, indexed in Pubmed: 25978372.

19. Lang R, Badano L, Mor-Avi V, et al. Recommendations for cardiac chamber quantification by echocardiography in adults: an update from the American Society of Echocardiography and the European Association of Cardiovascular Imaging. Eur Heart J Cardiovasc Imaging. 2015; 16(3): 233-271, doi: 10.1093/ehjci/jev014.

20. Peetz D, Schütt S, Sucké B, et al. Prognostic value of troponin T, troponin I, and CK-MBmass in patients with chronic renal failure. Med Klin (Munich). 2003; 98(4): 188-192, doi: 10.1007/s00063003-1243-3, indexed in Pubmed: 12715142.
21. Schmidt A, Stefenelli T, Schuster E, et al. Informational contribution of noninvasive screening tests for coronary artery disease in patients on chronic renal replacement therapy. Am J Kidney Dis. 2001; 37(1): 56-63, indexed in Pubmed: 11136168.

22. Conway B, McLaughlin M, Sharpe P, et al. Use of cardiac troponin $\mathrm{T}$ in diagnosis and prognosis of cardiac events in patients on chronic haemodialysis. Nephrol Dial Transplant. 2005; 20(12): 2759-2764, doi: 10.1093/ndt/gfi125, indexed in Pubmed: 16188899 .

23. Mavrakanas TA, Sniderman AD, Barré PE, et al. High ultrafiltration rates increase troponin levels in stable hemodialysis patients. Am J Nephrol. 2016; 43(3): 173-178, doi: 10.1159/000445360, indexed in Pubmed: 27064739.

24. Ellis K, Dreisbach AW, Lertora JL. Plasma elimination of cardiac troponin I in end-stage renal disease. South Med J. 2001; 94(10): 993-996, indexed in Pubmed: 11702827.

25. Daugirdas J, Depner T, Inrig J, et al. KDOQI Clinical Practice Guideline for Hemodialysis Adequacy: 2015 Update. Am J Kidney Dis. 2015; 66(5): 884-930, doi: 10.1053/j.ajkd.2015.07.015.

26. Voigt JU, Pedrizzetti G, Lysyansky P, et al. Definitions for a Common Standard for 2D Speckle Tracking Echocardiography: Consensus Document of the EACVI/ASE/Industry Task Force to Standardize Deformation Imaging. J Am Soc Echocardiogr. 2015; 28(2): 183-193, doi: 10.1016/j.echo.2014.11.003.

27. Galderisi M, Cosyns B, Edvardsen T, et al. Standardization of adult transthoracic echocardiography reporting in agreement with recent chamber quantification, diastolic function, and heart valve disease recommendations: an expert consensus document of the European Association of Cardiovascular Imaging. Eur Heart J Cardiovasc Imaging. 2017; 18(12): 1301-1310, doi: 10.1093/ehjci/jex244, indexed in Pubmed: 29045589.

28. Farsalinos KE, Daraban AM, Ünlü S, et al. Head-to-Head Comparison of Global Longitudinal Strain Measurements among Nine Different Vendors: The EACVI/ASE Inter-Vendor Comparison Study. J Am Soc Echocardiogr. 2015; 28(10): 1171-1181, e2, doi: 10.1016/j.echo.2015.06.011, indexed in Pubmed: 26209911.

29. Saenger AK, Beyrau R, Braun S, et al. Multicenter analytical evaluation of a high-sensitivity troponin T assay. Clin Chim Acta. 2011; 412(9-10): 748-754, doi: 10.1016/j.cca.2010.12.034, indexed in Pubmed: 21219893.

30. Fahim MA, Hayen AD, Horvath AR, et al. Biological variation of high sensitivity cardiac troponin-T in stable dialysis patients: implications for clinical practice. Clin Chem Lab Med. 2015; 53(5): 715-722, doi: 10.1515/cclm-2014-0838, indexed in Pubmed: 25527812 . 